\title{
Simple technique for measuring the energy-transfer-upconversion parameter in solid-state laser materials
}

\author{
J. W. Kim, I. O. Musgrave, M. J. Yarrow, W. A. Clarkson \\ Optoelectronics Research Centre, University of Southampton, Southampton, SO17 1BJ, UK
}

Scaling the output power from solid-state lasers to higher power levels, whilst maintaining high efficiency and good beam quality is a challenging task. The chief difficulty is heat generation and its deleterious effects on laser performance. The main origin of heat generation is often considered to be quantum defect heating. However, many solid-state laser media also suffer from the problem of energy-transfer-upconversion, which can lead to a dramatic reduction in the effective upper laser level lifetime and a large increase in the fractional heat loading unless appropriate measures are taken. Thus, an accurate knowledge of the upconversion parameter and the impact of upconversion on laser performance is very important for power scaling of solid-state lasers, particularly when operating in pulsed (Qswitched) mode. The standard method for determining upconversion parameters, based on measurements of fluoresecence decay times, can be quite time consuming and requires very careful attention to detail in the experimental set-up and pumping conditions if it is to yield accurate values.

In this paper we describe an alternative and easier method for measuring the upconversion parameter based on measuring laser threshold pump power as a function of resonator loss. This approach exploits the fact that upconversion loss is larger for higher excitation densities and that the excitation density in a laser can be increased by simply increasing the resonator loss. We have developed an analytical model for the dependence of threshold pump power, $\mathrm{P}_{\mathrm{pth}}$ on the upconversion parameter and the resonator loss for both four-level and quasi-three-level lasers. The resulting approximate expression for four-level lasers is simply:

$$
\mathrm{P}_{\mathrm{pth}} \approx \frac{\mathrm{hv} \mathrm{p}_{\mathrm{p}} \pi \mathrm{w}_{\mathrm{p}}^{2} \mathrm{~L}_{\mathrm{T}}}{2 \sigma \tau \eta_{\mathrm{q}}}\left(1+\frac{\mathrm{W}_{\mathrm{up}} \tau \alpha_{\mathrm{p}} \mathrm{L}_{\mathrm{T}}}{4 \sigma}\right)
$$

where $L_{T}=-\log _{e}\left(1-L_{R}\right)-\log _{e}(1-T)$ is the resonator loss parameter, $T$ is the transmission of the output coupler, $L_{R}$ is the round-trip resonator loss (excluding the output coupler transmission), $\mathrm{w}_{\mathrm{p}}$ is the pump beam waist radius, $\sigma$ is the emission cross-section, $\tau$ is the fluorescence lifetime of the upper laser level, $\eta_{\mathrm{q}}$ is the pumping quantum efficiency and $\alpha_{\mathrm{p}}$ is the absorption coefficient. The above expression assumes that the pump beam size is much larger than the TEM $\mathrm{oo}_{\mathrm{O}}$ size for the laser. Figure 1 shows measured values for threshold pump power for a typical $\mathrm{Nd} \mathrm{YVO}_{4}$ laser with a $\mathrm{Nd}^{3+}$ concentration of 1 at. $\%$ as a function of the resonator loss parameter. From this data, we obtained a value for the upconversion parameter (using equation (1)) of $1.5 \times 10^{-15} \mathrm{~cm}^{3} / \mathrm{s}$, which is in good agreement with a previously reported value $^{1}$. The corresponding expression for threshold in a quasi-three-level laser is rather more complicated, but can be used in a similar manner to determine the upconversion parameter. We have used this method to determine the upconversion parameter for a 1at.\% doped Er:YAG laser operating on the $1645 \mathrm{~nm}$ transition. Figure 2 shows the threshold pump power versus $\mathrm{L}_{\mathrm{T}}$. Analysis of the results yielded an upconversion parameter of $7.2 \times 10^{-18} \mathrm{~cm}^{3} / \mathrm{s} \mathrm{which} \mathrm{is}$ also in very close agreement with the value published in a previous report ${ }^{2}$. Further details of the threshold analysis and the advantages of this method for measuring upconversion parameters will be presented.

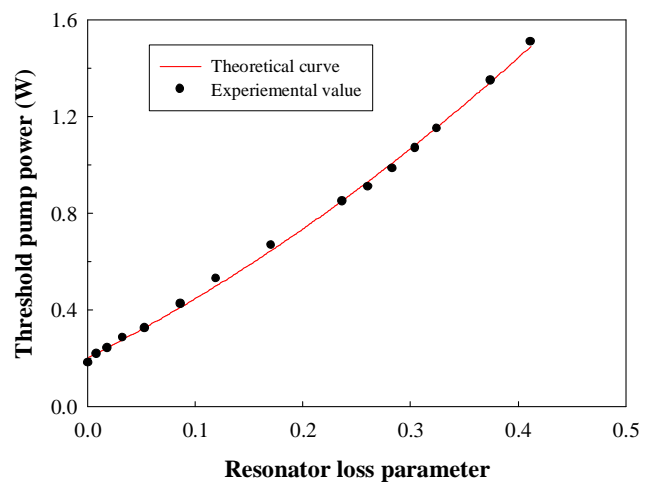

Fig. 1. Threshold pump power as a function of resonator loss in a $\mathrm{Nd}: \mathrm{YVO}_{4}$ laser.

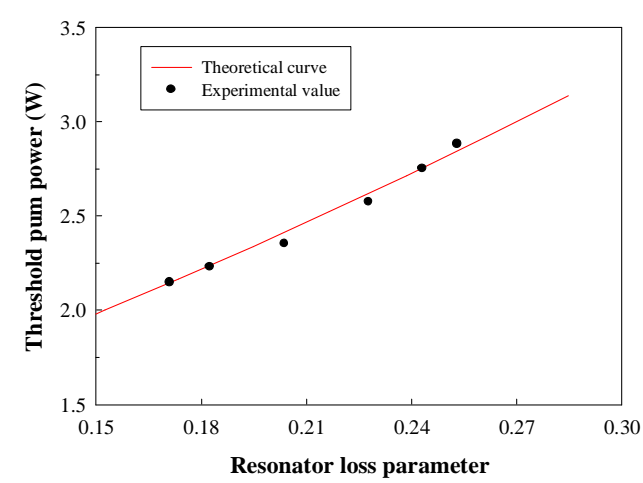

Fig. 2. Threshold pump power as a function of resonator loss in an Er:YAG laser.

1. Y. F. Chen, C. C. Liao, Y. P. Lan, and S. C. Want, "Determination of the Auger upconversion rate in fiber-coupled diode end-pumped Nd:YAG and Nd:YVO 4 crystals,", Applied Physics B 70, 487 (2000).

2. M. O. Iskandarov and A. A. Nikitichev and A. I. Stepanov," Quasi-two-level Er:YAG laser for the 1.6-mm range", J. Opt. Technol. 68, 885 (2001). 